Undergraduate Students, Health and Biomedical Sciences

\title{
Association between pre-existing conditions and hospitalization, intensive care services and mortality from COVID-19 - a cross sectional analysis of an international global health data repository
}

Basant M S Elsayed ${ }^{1}$, Lina Altarawneh1, Suhail A.R. Doi ${ }^{1}$, Tawanda Chivese ${ }^{1}$

1.Department of Population Medicine, College of Medicine, QU Health, Qatar University, Doha, Qatar

Objectives

- To investigate the association between pre-existing conditions and hospitalization, need for intensive care services (ICU) and mortality due to COVID- 19 .

\section{Introduction}

- Several studies have found an association between pre-existing comorbidities and worsening COVID-19 outcome. However, data on the effect of preexisting condition on outcomes from COVID19 in different age groups are scarce. Many of the published studies are small and have mainly come from High income countries. The scarcity of data from LMICs, where other socioeconomic determinants of poor outcomes from COVID-19, such as access to screening and treatment for the preexisting are less optimal.

- In this study, we used an international repository of cases with COVID-19 from 3 LMICs to examine the association between preexisting disease conditions and COVID-19 outcomes as it may help in prioritizing preventive measures such as vaccines, triaging patients in clinical settings and planning the care of patients in healthcare facilities.

\section{Methods}

- We used data on all cases recorded in the Global Health Data repository up to the 10th of March 2021.

- A cross-sectional analysis of associations between cardiovascular diseases, hypertension, diabetes, asthma and chronic pulmonary diseases and outcomes of hospitalization, ICU and mortality was done.

- We computed adjusted and unadjusted odds ratios (OR) of the three outcomes for each pre-existing condition in the ten-year age groups from 0-9 years and up to 110-120 years using multivariable logistic regression.

$$
\text { Fig } 1 \text { - Flow chart showing the study }
$$

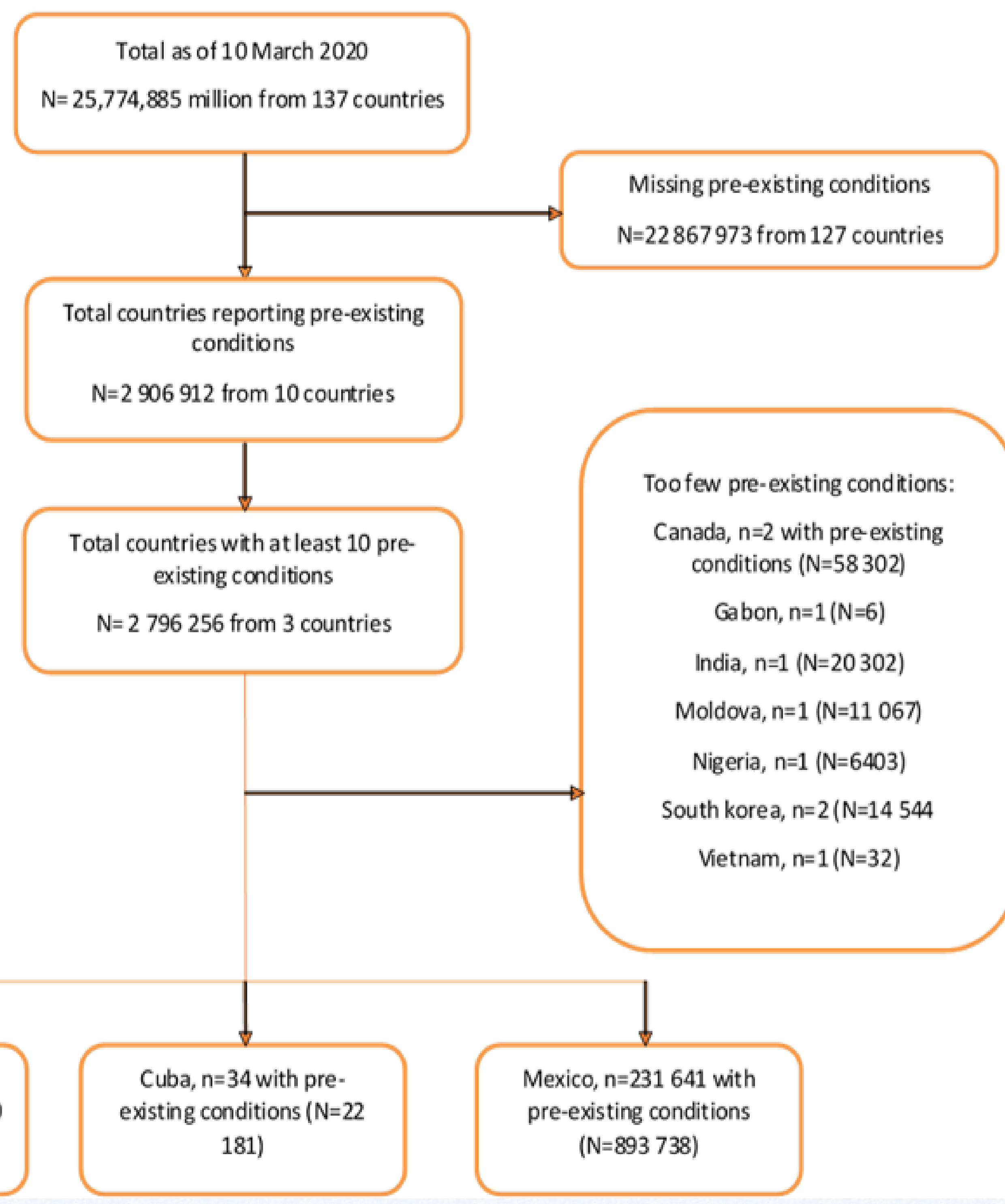

\section{References}

1. Silverio A, Di Maio M, Citro R, Esposito L, Iuliano G, Bellino M, et al. Cardiovascular risk factors and mortality in hospitalized patients with COVID-19: systematic review and meta-analysis of 45 studies and 18,300 patients. BMC Cardiovasc Disord. 2021;21(1):23.

2. Kumar A, Arora A, Sharma P, Anikhindi SA, Bansal N, Singla V , et al. Is diabetes mellitus associated with mortality and severity of COVID-19? A meta-analysis. Diabetes \& metabolic syndrome. 2020;14(4):535-45. 3. Figliozzi S, Masci PG, Ahmadi N, Tondi L, Koutli E, Aimo A, et al. Predictors of adverse prognosis in COVID-19: A systematic review and meta-analysis. Eur J Clin Invest. 2020;50(10):e13362.

4. Huang I, Lim MA, Pranata R. Diabetes mellitus is associated with increased mortality and severity of disease in COVID-19 pneumonia - A systematic review, meta-analysis, and meta-regression. Diabetes \& metabolic syndrome.

\section{Results}

A) Association between pre-existing conditions and hospitalization due to COVID-19 among the different age groups.

- The overall adjusted odds of hospitalization for each pre-existing condition were; CVD (OR 1.7, 95\%CI 1.7-1.7), hypertension (OR 1.5, 95\%CI 1.4-1.5), diabetes (OR 2.2, 95\%CI 2.1-2.2), obesity (OR 1.7, 95\% CI 1.6-1.7), kidney disease (OR 5.5, 95\%CI 5.2-5.7) and lung disease (OR 1.9, 95\% CI 1.8-1.9). The odds of hospitalization were higher in children and young adults who had this comorbidities. Of note, kidney diseases were associated with almost 16 folds increase in hospitalization rate among young patients (10-19 yrs) compared to those with no kidney diseases.

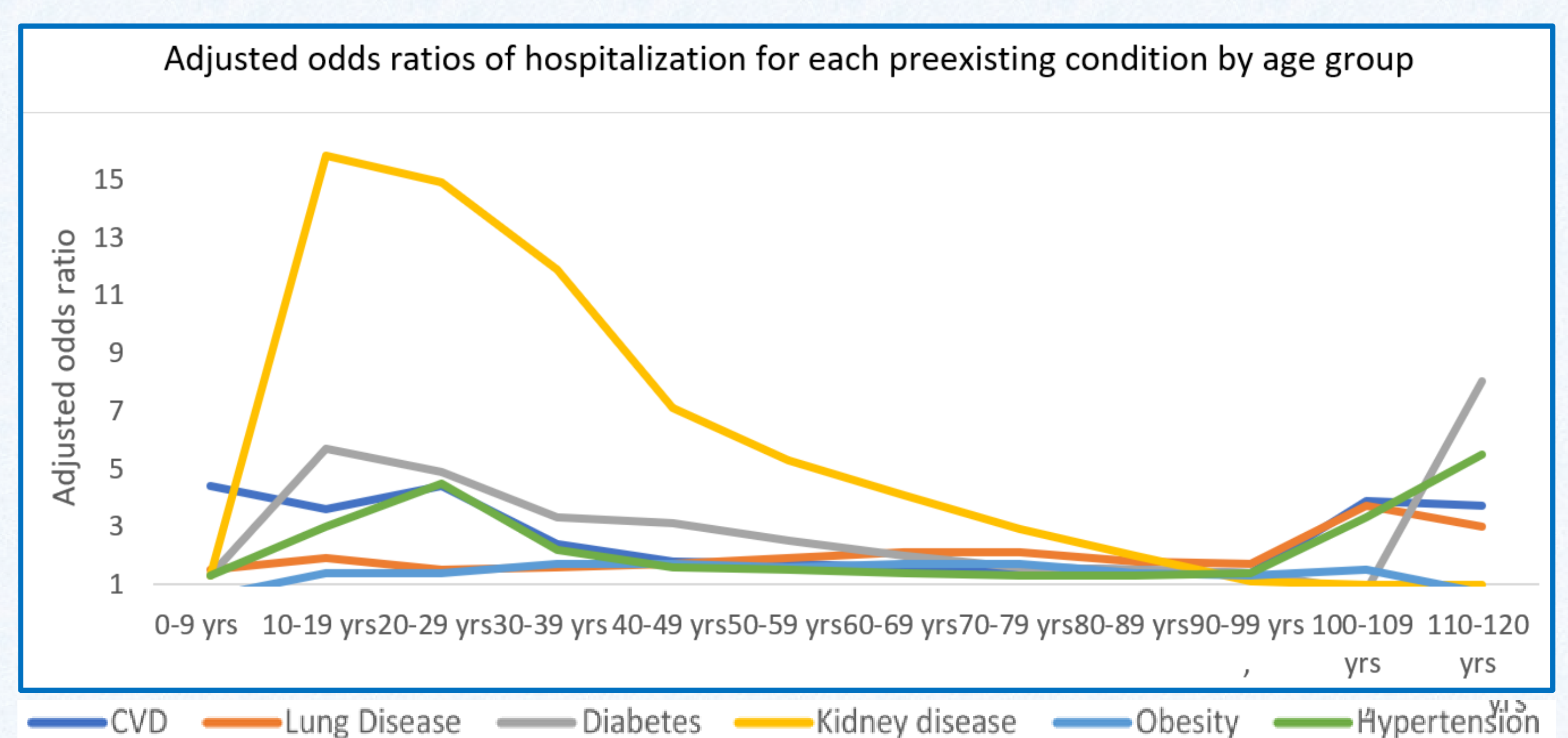

B) Association between pre-existing conditions and ICU admission due to COVID-19 among the different age groups.

- The overall adjusted odds of ICU for each pre-existing condition were; CVD (OR 2.1, 95\%CI 1.8-2.4), hypertension (OR 1.3, 95\%CI 1.2-1.4), diabetes (OR 1.7, 95\%CI 1.5-1.8), obesity (OR $2.2,95 \%$ CI 2.1-2.4), kidney disease (OR 1.4, 95\%CI 1.2-1.7) and lung disease (OR 1.1, 95\%CI 0.9-1.3). The odds of ICU admission were higher in younger patients having comorbidities compared to older patients with the same comorbidies. Of note, diabetes was associated with almost 10 times the odds of ICU admission among young adults (20-29 yrs) compared to those with no diabetes. In addition, kidney diseases had 5 times the odds of ICU admission compared to those with no kidney diseases.

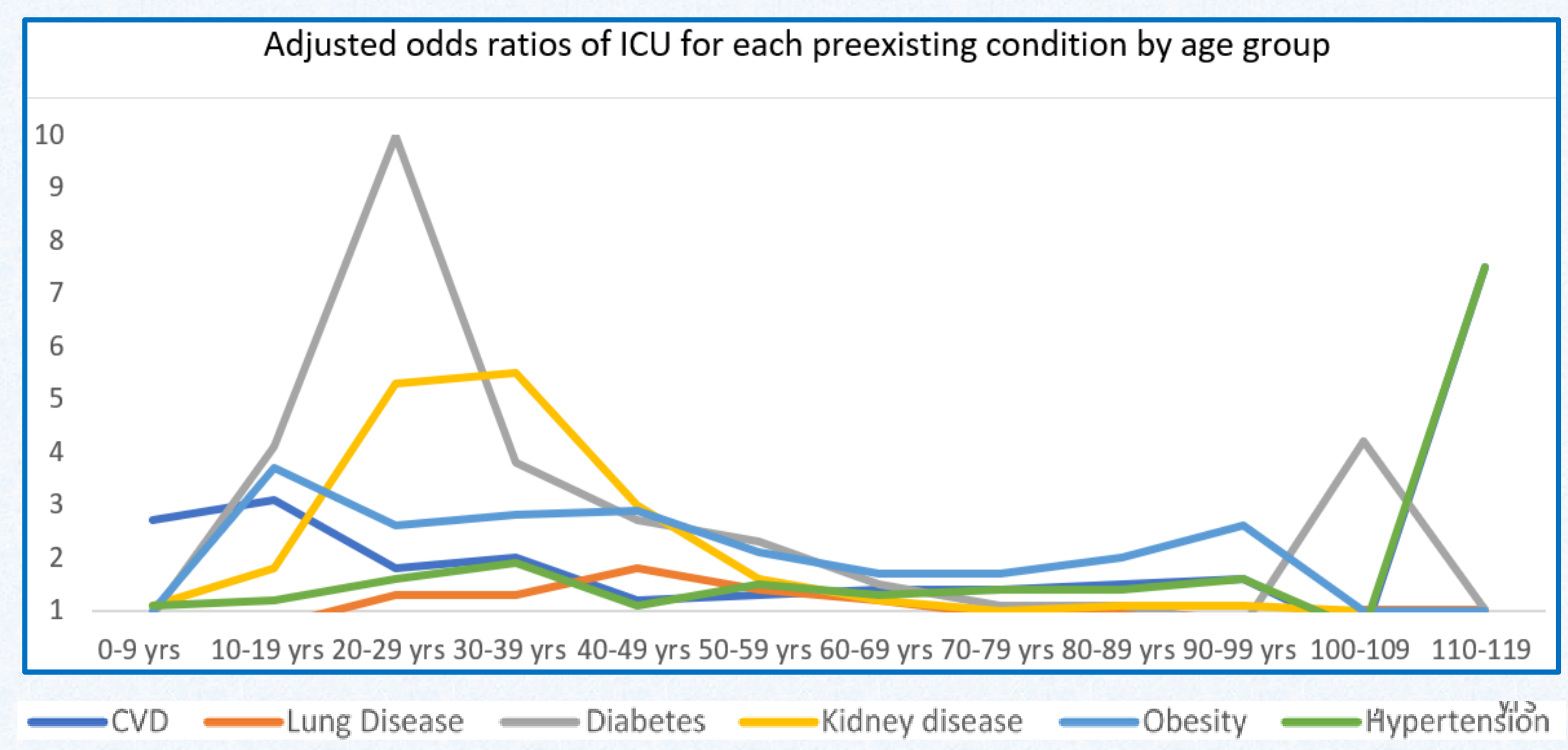

C) Association between pre-existing conditions and mortality due to COVID-19 among the different age groups.

- The overall adjusted odds of mortality for each pre-existing condition were; CVD (OR 1.7, 95\%CI 1.6-1.7), hypertension (OR 1.3, 95\%CI 1.3-1.4), diabetes (OR 2.0, 95\%CI 1.9-2.0), obesity (OR 1.9, 95\%CI 1.8-2.0), kidney disease (OR 2.7, 95\%CI 2.6-2.9) and lung disease (OR $1.6,95 \%$ CI $1.5-1.7)$. The odds of mortality were higher in younger patients having comorbidities compared to older patients with the same comorbidies. Of note, kidney diseases increase the odds of ICU admission by almost 21 folds among young adults (20-29 yrs) compared to those with no kidney diseases.

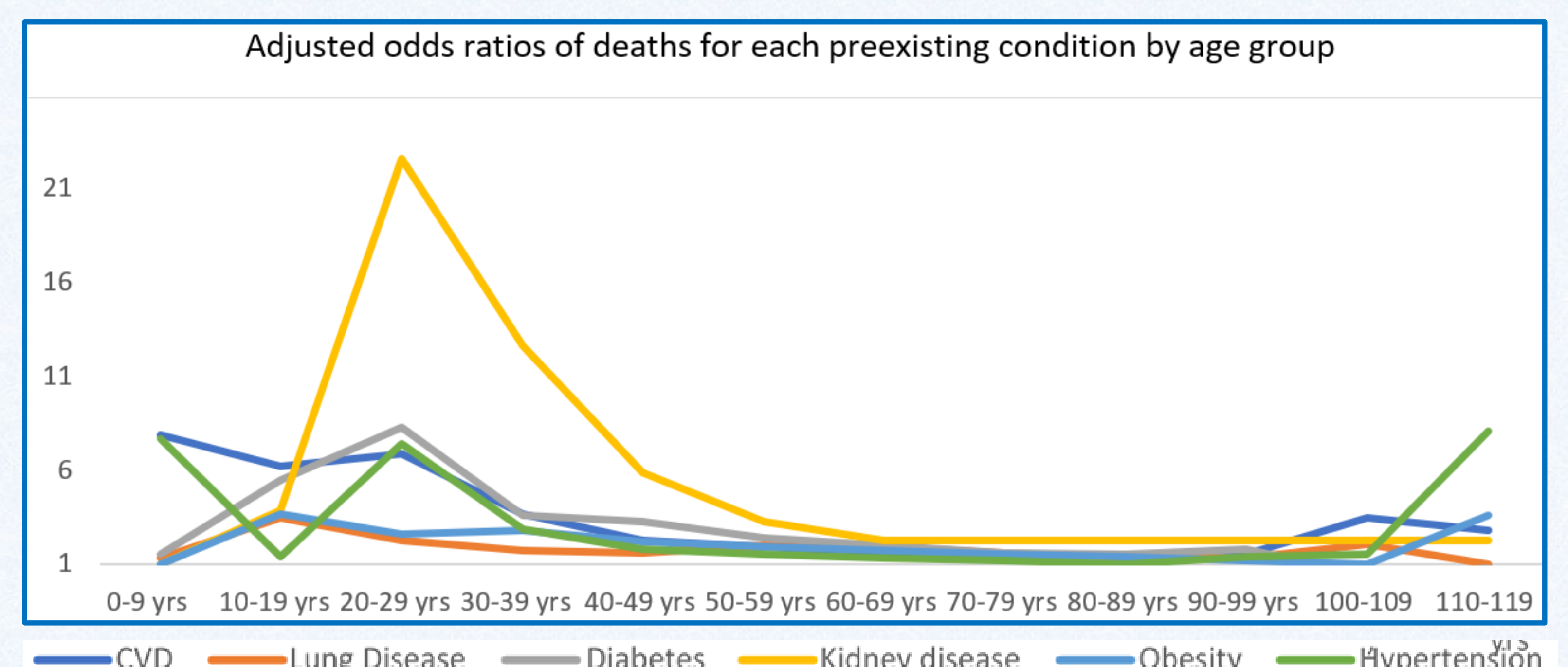

Conclusion

- Individuals with CVD, hypertension, diabetes, obesity, lung diseases and kidney diseases have high odds of hospitalization, ICU and mortality from COVID-19. The odds of these outcomes are especially elevated in children and young adults with these preexisting conditions. 